\title{
Patient Infusion Pattern based Access Control Schemes for Wireless Insulin Pump System
}

\author{
Xiali Hei, Member, IEEE, Xiaojiang Du, Senior Member, IEEE, Shan Lin, Senior Member, IEEE, \\ Insup Lee, Fellow, IEEE, and Oleg Sokolsky, Member, IEEE
}

\begin{abstract}
Wireless insulin pumps have been widely deployed in hospitals and home healthcare systems. Most of them have limited security mechanisms embedded to protect them from malicious attacks. In this paper, two attacks against insulin pump systems via wireless links are investigated: a single acute overdose with a significant amount of medication and a chronic overdose with a small amount of extra medication over a long time period. They can be launched unobtrusively and may jeopardize patients' lives. It is very urgent to protect patients from these attacks. We propose a novel personalized patient infusion pattern based access control scheme (PIPAC) for wireless insulin pumps. This scheme employs supervised learning approaches to learn normal patient infusion patterns in terms of the dosage amount, rate, and time of infusion, which are automatically recorded in insulin pump logs. The generated regression models are used to dynamically configure a safe infusion range for abnormal infusion identification. This model includes two sub models for bolus (one type of insulin) abnormal dosage detection and basal abnormal rate detection. The proposed algorithms are evaluated with real insulin pump. The evaluation results demonstrate that our scheme is able to detect the two attacks with a very high success rate.
\end{abstract}

Index Terms—-wireless insulin pump, implantable medical devices, access control, infusion pattern, patient safety.

\section{INTRODUCTION}

To help 25.8 million Americans [1] with diabetes, a growing number of wireless and wired insulin pumps have been used by diabetic patients to deliver insulin into their circulatory systems. Wired insulin pumps are used by nurses directly without wireless USB. In 2005, there were about 245,000 wireless pump users, with this market expected to grow $9 \%$ annually between 2009 and $2016[2,3]$. It is very important that these wireless insulin pumps are reliable, secure, and safe.

Unfortunately, most of the existing wireless insulin pumps lack sufficient security mechanisms to protect patients from malicious attacks and overdose incidents. For example, a pump malfunction has caused a patient's death [4], where the pump went into the PRIME function when the patient was asleep and delivered the entire cartridge of insulin. Insulin pumps have preset minimum and maximum dosage levels as well as infusion rates, which is required by the FDA [5]. However, researchers have shown that these levels could be remotely disabled

- X. Hei is with the Department of Computer Science and Information Technologies, Frostburg State University, Frostburg, MD 21532, USA. E-mail: xhei@frostburg.edu.

- X. Du is with the Department of Computer and Information Sciences, Temple University, Philadelphia, PA 19122, USA. E-mail: dux@temple.edu.

- S. Lin is with the Electrical and Computer Engineering Department, Stony Brook University, Stony Brook, NY 11790, USA. E-mail: shan.x.lin@stonybrook.edu.

- I. Lee and O. Sokolsky are with the Department of Computer and Information Science, University of Pennsylvania University, Philadelphia, PA, 19104 USA e-mail: \{lee, sokolsky\}@cis.upenn.edu.

We would like to Min Xiao for comments. This work was supported in part by the US NSF under grants CNS-0963578, CNS-1022552, CNS-1065444, IIS-1231680, CNS-1239108, as well as CNS-1035715. by attackers [6]. Without this basic protection mechanism, the insulin pump is vulnerable to several attacks. In this paper, we investigate two new fatal attacks that are specifically targeted at wireless insulin pumps. The first type of attack is a single acute overdose attack: the attacker can issue a one-time overdose (underdose) containing a significant amount of medication to a patient. For diabetic patients, the effects of the insulin overdose can include dizziness, drowsiness, and nausea, ultimately leading to seizures, coma, and in the worst case death [7]. The second type of attack is chronic overdose (underdose) with an insignificant amount of medication being delivered over a long period, e. g. months. The chronic overdose of insulin can directly cause low blood glucose (BG), which leads to various complications and is extremely difficult to detect. Given that this attack can be performed even without modifying the insulin pump settings, it is exceptionally challenging to defend against.

For patients' safety, it is necessary to defend against these two types of attacks on insulin pumps. Many wireless insulin pumps, e. g. Medtronic MiniMed 512, automatically record detailed information about each infusion in its $\log$ file. Figure 1 shows an insulin dosage example in a day. We consult 10 patients and several doctors, analyze 10 patients' data. The detailed information includes the infusion rate, dosage, BG level, patient id, and time of day for each infusion. Given this information, we observe normal infusion patterns for home care diabetic patients. Thus, we propose a novel access control mechanism using a supervised learning method. To learn these normal patterns, the regressions are designed to analyze infusion dosage history and predict future infusion dosages. Once data collected and analyzed after 
3 months, our scheme can generate a safety range for a specified time interval. The automatically recorded log data are raw data, which are encrypted and stored on the pump. The encryption key is applied to standard authentication. An attacker needs to encrypt the data and decode the raw data to get meaningful records, which is costly. During the data collection period, we use the naive solution in Section 7.1 to protect the patient.

Our access control algorithm design has three unique features: 1) this algorithm utilizes the temporal correlation of infusions for any particular patient. Patient specific infusion patterns are captured over time, and the long term changes of infusion patterns can also be detected; 2) a safety range is dynamically updated at different times of the day based on the online model, (the safety range counts for the in-situ variations of the insulin injection); and 3) this algorithm doesn't require any extra information, all the data required is already present in the insulin pump logs. Furthermore, the linear regression design requires little memory and computing capacity, which can be done in real-time even on resource limited computing platforms. Our algorithm can also identify infusion mistakes made by doctors or patients, such as an erroneous dosage input. In emergency situations, insulin pump users should be allowed to infuse a larger than usual dosage. Several bio-metric based solutions have been proposed to address this problem in emergency, which is not the focus of this paper. Several bio-metric based solutions using EKG or ECG are not suitable for insulin pumps.

There are a number of prior works on implantable medical devices. These works provide valuable research results for our study. For example, with a pump serial number (SN) and USB device easily purchased from eBay, Radcliffe was able to track data transmitted from the computer and control the insulin pump's operations [9]. Radcliffe was also able to cause BG management devices to display inaccurate readings by intercepting wireless signals sent between the sensor device and the management device. Kevin Fu et al. detailed how to reprogram an implantable cardiac defibrillator remotely, causing the victim to receive a malicious shock [10]. Measurements in another paper [29] show that in free air, intentional EMI under $10 \mathrm{~W}$ can inhibit pacing and induce defibrillation shocks at distances up to $1-2 \mathrm{~m}$ on implantable cardiac electronic devices. Additionally, harvesting patient data in the region is easily executed via an eavesdropping attack. Previous literature [8] has analyzed these possible attacks and has proposed using a traditional cryptographic approach (rolling code) and body-coupled communication to protect the wireless link and insulin pump system. However, these proposed solutions do not address the overdose attacks that are studied in this paper. Also, the authors of this paper did not decode the Carelink USB driver. In this paper, we present a novel supervised learning based approach for insulin pump access control. It includes two bolus abnormal dosage detection models, one updated basal (another type of insulin) abnormal rate detection model and one algorithm to combine them. Note that, to save the resources, we assume all the near optimal parameters are obtained through offline learning. Offine learning is downloading the data, preprocessing the data, getting the optimal parameters through running the generic algorithms on the laptop or PC instead of pumps, and building the normal dosage model. Then we use these parameters in online regression and detection. We also update the parameters according to the update policies in Section 5.2.3 and 5.3.2. To do this, we redo the pattern learning and get the optimal parameters and update the detection models running on the pumps.

Our solution is evaluated with real insulin pump logs obtained from Medtronic pumps including MiniMed 511, 512, 522 and Paradigm Revel 723 in home care systems for diabetic patients. Several log files are tested. Each $\log$ file contains the infusion records of a particular patient for up to 6 months. We use a cross-validation approach to tune our model. The first $80 \%$ of logs are selected as a training data set, and the remaining $20 \%$ are used for testing. Malicious attacks are simulated in combination with the normal infusions. Evaluation results show that our algorithm can effectively identify the single overdose attack with a success probability up to $98 \%$ and detect the chronic overdose attack with an about $100 \%$ success rate. Our contributions are summarized as follows:

- A novel personalized patient infusion pattern based access control scheme for wireless insulin pump is proposed. To the best of our knowledge, we are the first group to utilize patient specific infusion patterns to identify malicious overdose attacks on insulin pumps. Our work is able to prevent the malfunction of nurses and patients as well. Also, our scheme has close-loop properties.

- Our solution dynamically calculates a safety dosage range at different times based on the online learning model. A simplified bolus abnormal dosage detection is presented, with high efficiency and low energy consumption.

- Experimental results with real insulin pump data sets demonstrate that our solution can defend against the overdose attacks effectively with a success rate above $98 \%$.

The remainder of this paper is organized as follows: In Section 2 we describe the background and attack models. We analyze patient infusion patterns in Section 3. In Section 4 we present the detailed patient infusion pattern based access control scheme. We describe our real experimental results in Section 5. We extend our scheme in Section 6. We show related discussions in Section 7. In Section 8, we review the related work, and we conclude the paper and discuss the future work in Section 9. 


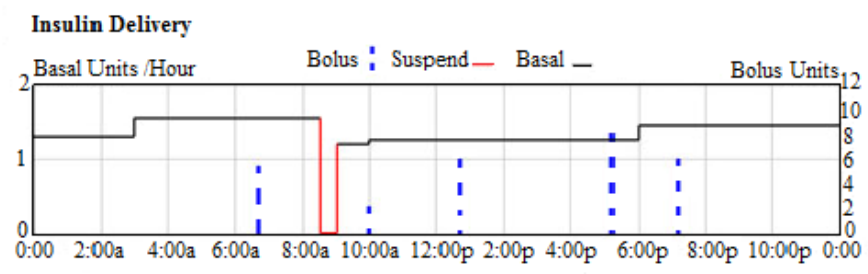

Fig. 1. Daily insulin dosage example of a patient used with permission from Medtronic Mini-Med, Inc.

\section{System and Attack Models}

\subsection{Background and System Model}

An infusion pump infuses fluids or medicine into a patient's circulatory system. Wireless insulin pumps are widely used to deliver insulin into a diabetic patient's body to treat diabetes. An insulin pump usually delivers a single type of rapid-acting insulin in two ways:

- A bolus dose that is pumped to cover food eaten or to correct a high BG level.

- A basal dose that is pumped continuously at an adjustable basal rate to deliver insulin needed between meals and at night.

It is the responsibility of the pump user to start a bolus or change the basal rate manually. The patient set the bolus dosages according to the algorithm builtin the pump and basal rate suggested by the doctors. The bolus dosages and basal rates could be changed through Carelink USB by doctor or nurses or attackers. The times of delivering bolus in a day depend on the patient's behavior. Figure 1 shows the insulin infusion record for a diabetic patient over 24 hours. As illustrated in the figure, the insulin basal rates are slightly different during different time periods within one day. The basal rate is a continuous infusion that lasts for 24 hours. The infusions with the bolus dosages are discrete and occur around $7 \mathrm{am}, 10 \mathrm{am}, 12 \mathrm{pm}, 5 \mathrm{pm}$, and $7 \mathrm{pm}$ each day. The bolus dosages have three categories: normal, square, and dual. Patients choose a type of bolus with specified amounts of dosages. The diabetic individual usually delivers a square bolus or a normal bolus at a fixed time interval that accounts for breakfast, lunch, dinner, and other cyclical events throughout the day. Factors such as carbohydrate (carb) ratio, insulin sensitivity, and target high BG are typically unique to each patient.

Figure 2 shows the components of a Medtronic Paradigm real-time insulin pump system. The OneTouch meter obtains BG readings from the patients' finger prick tests. The BG level is transmitted from the OneTouch meter to the insulin pump via the wireless link 2. The sensor tests the glucose trend (up or down) in patients' interstitial fluid. And it sends the trend to continuous glucose monitor system via wireless link 3 and to pump via wireless link 6 . Wireless links 2 and 6 use similar protocol and suffer the same attacks. The insulin pump delivers insulin to the patient. The remote control unit is operated by the user to send instructions (such as suspend and resume basal rate) to the insulin pump via wireless link 1. Wireless link 4 and 7 transmit historical glucose readings to a USB device that uploads the information to a web service. Wireless link 5 allows the Carelink USB device to gather reports on BG trends and patterns. Wireless link 6 sends current glucose levels to the pump. A laptop or PC is utilized by the Carelink USB device to upload data to a web-based management system.

\subsection{Overdose Attack over Wireless}

Given the wireless insulin pump system, we discuss potential attacks. To connect two components, a user must manually enter the $\mathrm{SN}$ of that component being wirelessly connected. Once all of the wireless connection$\mathrm{s}$ among components are established, the insulin pump can display BG readings from sensors and adjust the bolus dosage and basal rate according to control unit commands.

The wireless communication in the system is not encrypted. As a result, attackers can easily compromise the wireless links in this system. Various malicious actions can be conducted after the wireless links are compromised. For example, attackers can display incorrect BG readings on the insulin pump via link 2 . We refer to this attack as Radcliffe's attack. Another attack is that an attacker suspends the basal rate delivery using link 1. We do not discuss this attack in our paper because it can be easily noticed by patients.

Insulin pump users can modify the pump settings using the Carelink Pro software on a computing device, such as a laptop. The new settings are uploaded to the pump using the attached Carelink USB device via wireless link 5. In this case, attackers may use customized software and a wireless sniffer to obtain the SN of all pumps within 300 feet, and can, therefore, compromise wireless link 5 to change the settings of the pump without being noticed. Using this security flaw, an attacker can 1) disable the alarms of the pump, 2) change the maximum allowable dosage of the pump, and 3) deliver a fatal dose to the insulin pump user. The delivery of a lethal dose is life-threatening and must be defended against.

In this paper, we focus on the attacks that are based on the compromised wireless link 5. Particularly, we focus on two types of attacks related as follows,

- Single acute overdose. This attack issues a one-time overdose (underdose) to the patient. A significant amount of medication that is larger (less) than the normal dosage will be delivered to the patient using the insulin pump in a short period. Given that a dosage of this magnitude is fatal, it is critical to prevent this attack.

- Chronic overdose. This attack issues extra portions of medication to the patient over a long period, e. g. weeks or months. One or two instances of 


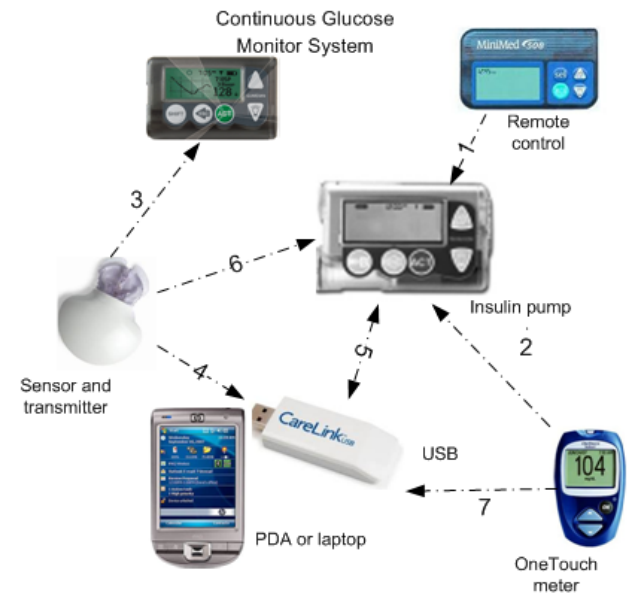

Fig. 2. A real time insulin pump system used with permission from Medtronic Mini-Med, Inc.

the small overdose are not critical; however, such overdoses for a long period can put the patient's life in danger. It is the attack could not be identified by one-time check. The clinicians and patients may not notice the small amount of overdose, since it does not cause obvious symptoms until the dosages have accumulated to a dangerous level. This can also cause various complications to the patient. It can be defend against because we use blood glucose (BG) as a parameter. It works as a close-loop feedback to another parameter such as EB (expected bolus). When there is a chronic underdose attack, the BG will be higher and the EB will be larger. So the increased EB will correct such a chronic underdose attack.

The authentication scheme is crucial. However, the existing authentication with a code is not secure if an attacker can get close enough. Right now, there are no authentication schemes over wireless link 5 . In this paper, we assume the wireless link 5 has a standard authentication scheme and the patient's parameters can only be changed manually. The devices only provide the interface to set the parameters manually. The system utilizes standard authentication protocol ISO 9798-2.

\section{DATA ANALYSIS}

Our study is based on real insulin pump records provided by anonymous diabetic patients. Over the last year, several patients used Medtronic wireless insulin pumps at their homes for at least three months. All those patients were able to upload their infusion log data to the Carelink online management system.

\subsection{Infusion Record Analysis}

Since the dataset was real patient data in a format proprietary to Medtronic Inc., a substantial effort was required to clean the data. First of all, many of the recorded events are not directly related to the delivered patients' dosages. Secondly, there is a time difference between the time of recording features that we used in Section 3.3 and 3.5 and the programming of doses. We preprocess this data by an automation tool to make it suitable for analysis. This tool retrieves the feature set for each record related to the basal rate and bolus, adds a label, and normalizes the feature, and classifies it into basal rate logs and bolus logs. Then, we count the occurrences of each bolus according to the time label on each day. We also calculate the total dosage of bolus during each period $\Delta$, which starts when the patient requires a bolus. The mean $E$ and standard deviation $\sigma$ of daily total insulin were calculated, as well.

From the preprocessed data set, we find that the estimated bolus dose and other nine variables (BG level, active insulin and insulin sensitivity etc.) were correlated. There are a few other variables (e. g. the amount of exercise) that are known to affect the estimated bolus level. Unfortunately, we do not have access to this data.

We explore the infusion records of patient A over the course of several days. We observe that, during breakfast time, there are 1-2 bolus doses; during lunch time, there are 1-3 bolus doses; during dinner time, there are also only 1-2 bolus doses. Figure 3 is a histogram of patient A's daily bolus dosage in 3 months. It shows that the bolus delivery was highly aligned with the time of the day. We also perform the Shapiro-Wilk test for all the patients' total daily insulin. The test result shows that they all obey normal distribution because all of the $p \mathrm{~s}$ of them are greater than 0.05 . These results suggest that the mean of daily total insulin dosage of a patient was stable over the treatment period. For example, Figure 4.a is a histogram of patient A's total daily insulin for 3 months, which indicates to us it may follow the normal distribution. In Fig. 4.a, the y-axis is the number of days having a special total daily insulin. Figure 4.b shows the mean $E$ and 2-standard deviation $2 \sigma$ of patient A's total daily insulin in 3 months. We can see that they are bounded within $[E-2 \sigma, E+2 \sigma]$. We plot the histogram and probability of the 3-month total daily insulin dose. In Figure 4.c, the number represents the number of days having a special total daily insulin. The unit of the above subfigure is percent. And it shows that the $99.5 \%$ of daily total insulin falls in the range [22-44].

\subsection{Patient Insulin Dosage Pattern 1}

From the study results, we observe that there generally exist patterns of bolus and basal rate infusions, even though each patient may have his/her own circadian rhythm. A patient's eating habits can be manifested from various factors, including their profession, diet, exercise routine, degree of insulin sensitivity, or a host of other factors. However, there are five main periods related to the infusion. These are breakfast, lunch, dinner, evening, and the time when the patient is asleep. A patient can choose a preferred time for infusion during one of these time periods. Typically, a patient requires a high insulin dose in the morning, and less around 4-6pm, 

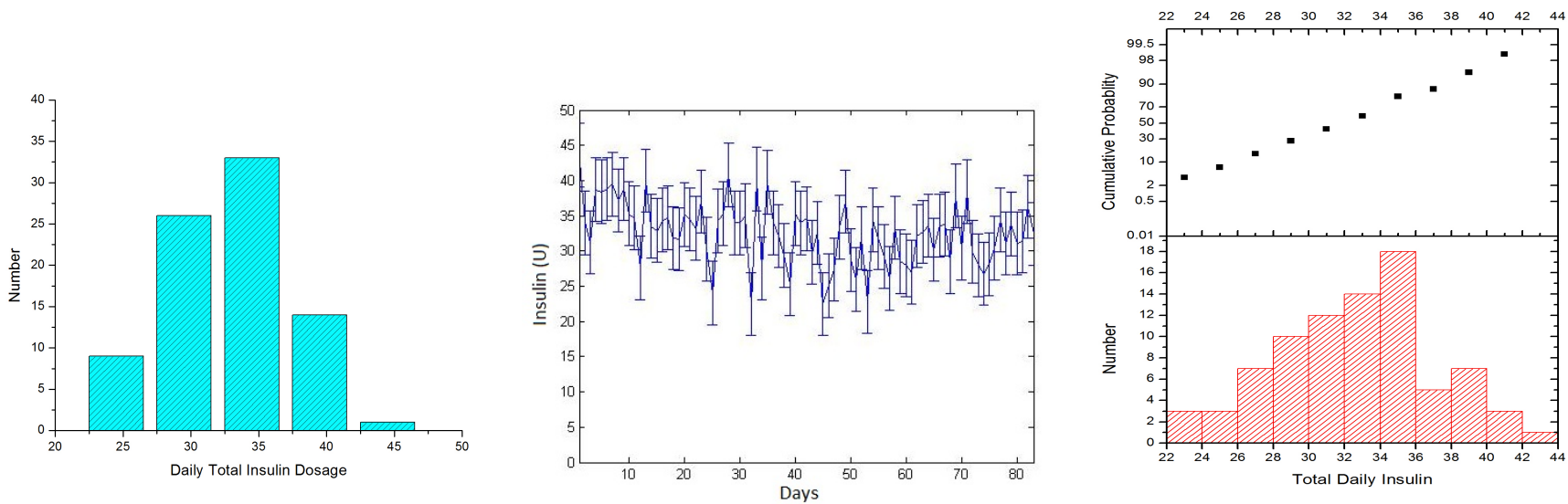

(a) The histogram of patient A's daily total (b) The $2 \sigma$-errorbar of patient A's daily total (c) The histogram and probability of patient A's insulin dosage in 3 months insulin dosage

daily total insulin dosage in 3 months

Fig. 4. Patient A's daily total insulin dosage analysis

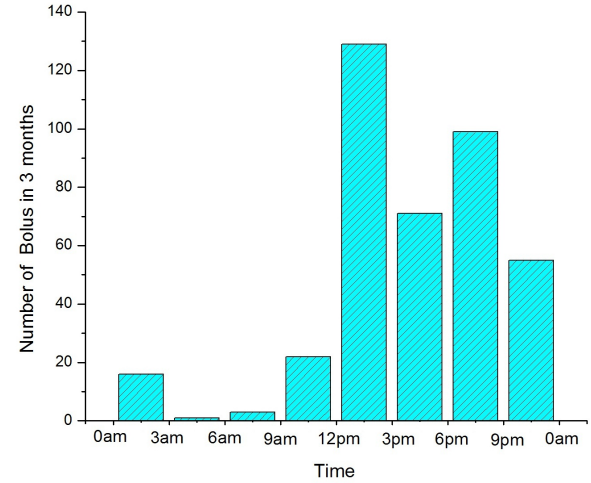

Fig. 3. The histogram of patient A's daily bolus dosage in 3 months

then more after 12am to counter regulatory hormones during the night. Different patients also exhibit different peak times of BG levels. Although there are exceptions preventing adherence to a rigorous schedule, the patient still has his/her own schedule pattern based on the functions that the insulin pump can provide. We reasonably believe that each patient exhibits a pattern that is distinguished enough that it may be used to identify abnormal events.

\subsection{Bolus Data Feature Set 1}

Our assumption is that the history records are helpful in the prediction of the insulin dosage of the same patient. This is true for 10 patients we have consulted because that a diabetes tends to have a strict meal plan and the parameters stored in the pump are suggested by a doctor and seldom changed. Having this in mind, we extract related features. The features we consider to be relevant to our regression model are: Time, Estimate Bolus, Target High BG, Target Low BG, Carb Ratio, Insulin Sensitivity, Carb Input, BG Input, Correction Estimate, Food Estimate, Active Insulin, Daily Total Insulin, Basal Pattern
Name, Index, Basal Rate, and Start Time. All of these features are expected to have a strong correlation with the timestamps of the records. We will use some of them in our detection models.

\subsection{Patient Bolus Dosage Pattern 2}

Reexamining the patient data, we observe that generally the bolus dosage selected $(B o)$ equals the estimated bolus $(E B)$ if the patient uses the bolus wizard function, even though each patient may have his/her own circadian rhythm. If the patient doesn't use the bolus wizard function, he/she seldom has bolus doses or the dosage he/she selects is stable. Some patients using bolus wizard may adjust the $B o$ according to the estimated bolus $E B$; however, the adjustment behavior has patterns. The total number of adjustments in a day is less than 2 times. Also, if the patient makes a positive $(B o-E B)$ several times continually, he/she usually makes another negative $(B o-E B)$ to balance the increasing insulin behavior. As we observed, the total adjustment dosage $\sum(B o-E B)$ in a day has a threshold unless the patient eats a lot during an event, exercises a lot or is sick. Based on the functions that the insulin pump can provide, the patient has no way to avoid the algorithm embedded and control the time a dosage used. If the bolus type is not "Normal", the total $(B o-E B)$ in a small window is supposed to be 0 because the patient wants to split a big $E B$ into several small bolus dosages. Otherwise, we think the total $(B o-E B)$ in a small window is not 0 is an adjustment event. Besides, the adjustment range has a threshold.

\subsection{Bolus Data Feature Set 2}

We find that a patient knows his body and the patient has a unique psychological behavior during the adjustment process through our data analysis. This information is helpful in the prediction of the insulin dosage 
of the same patient. This is true for the 10 patients we analyze. Having this in mind, we extract related features. The features we consider to be relevant to our second bolus dosage abnormal detection model are Time, Estimate Bolus, Insulin Sensitivity, Bolus Dosage Selected, and Date. The composite feature $(B o-E B)$ is expected to have a strong correlation with the timestamps of the records. We will use some of them in our detection models.

\section{Details of PIPAC Scheme}

In this Section, we present our access control scheme in detail. If our model returns "Fail", the dosage will not be accepted by the insulin pump, and an alarm will be issued to the patient, as well. Our scheme can defend against the two kinds of attacks that we have outlined.

\subsection{Overall Detection Model}

The goal of our scheme is to identify abnormal infusions of bolus dosage, basal rate, and total daily insulin. Table 1 summarizes the notations used in the rest of the paper. Our scheme includes two bolus abnormal dosage detection models, one updated basal abnormal rate detection model and one algorithm to combine them. Figure 5 illustrates the total abnormal dosage detection process. Firstly, at reset time (generally at $0 \mathrm{am}$ ), we check whether the total daily insulin falls in the safety range. If it falls out safety range, we send an alarm to the patients. Otherwise, we check the operation type. If it is bolus dosage, we check whether the vector has $E B$ feature. If "Yes", we can choose bolus abnormal dosage detection model 1 or 2 to monitor the bolus dosage. Otherwise, we can use abnormal dosage detection model 1 to detection the abnormal bolus dosage. If it is a basal rate, we adopt the abnormal basal rate detection model to monitor it in real time. For all above detections, if the dosage pass the detection, we will continue the safe dose. Otherwise, we block the dose and alarm the patient.

We use the Mean Squared Error ( $M S E$ ) to measure the performance, which is given in equation (1). The error is the difference between the estimated value and the real value, where $\bar{m}$ is the test sample size.

$$
M S E=\frac{1}{\bar{m}} \sum_{i=1}^{\bar{m}}\left(f\left(u_{i}-v_{i}\right)\right)^{2} .
$$

$$
\begin{aligned}
& S C C^{2}= \\
& \frac{\left(\bar{m} \sum_{i=1}^{\bar{m}}\left(f\left(x_{i}\right) y_{i}\right)-\sum_{i=1}^{\bar{m}} f\left(x_{i}\right) \sum_{i=1}^{\bar{m}}\left(y_{i}\right)\right)^{2}}{\left(\bar{m} \sum_{i=1}^{\bar{m}} f\left(x_{i}\right)^{2}-\left(\sum_{i=1}^{\bar{m}} f\left(x_{i}\right)\right)^{2}\right)\left(\bar{m} \sum_{i=1}^{\bar{m}} y_{i}^{2}-\left(\sum_{i=1}^{\bar{m}} y_{i}\right)^{2}\right)}
\end{aligned}
$$

The squared correlation coefficient $(S C C)$ is the predictive percent of behavior in the output that can be explained by the input. If the $S C C$ value is between $70 \%$ to $100 \%$, it is considered to have a strong relationship. By any regression method, we only can predict a value. Instead, we want to obtain a safety range. According to

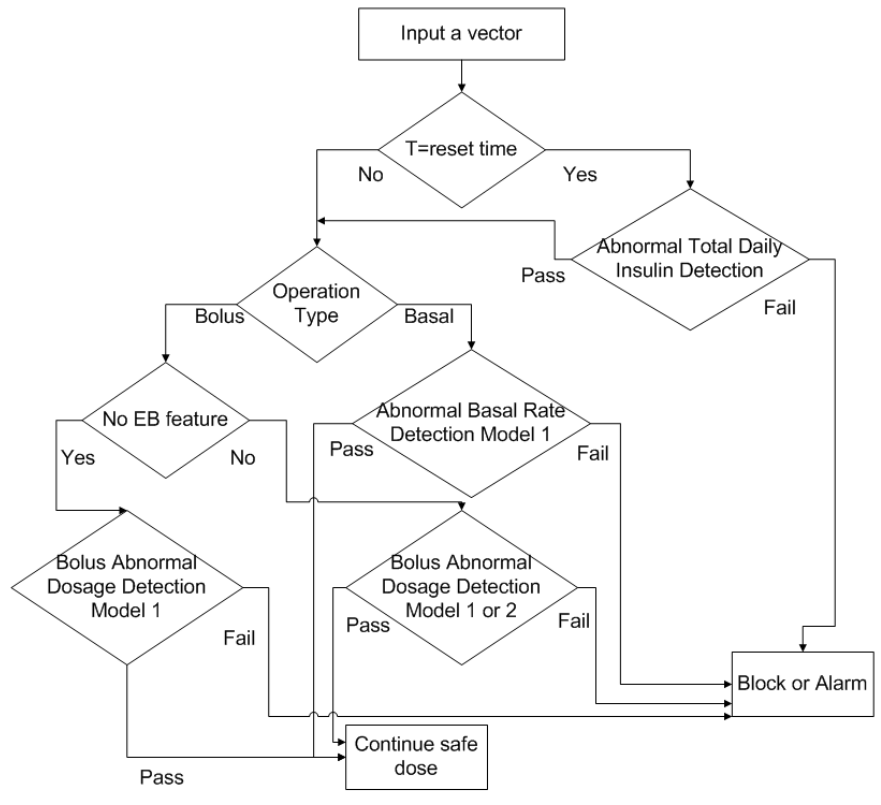

Fig. 5. Abnormal dosage detection process

TABLE 1

Notations description in PIPAC scheme

\begin{tabular}{|c|c|}
\hline Notation & Description \\
\hline Bolus $_{p}$, Basal \\
$C B, B o$ & Predicted Bolus and Basal rate \\
& Cumulative bolus dosage from $\Delta_{s t}$, \\
$\Delta, \Delta_{s t}$ & Bolus dosage to be checked \\
$S R_{l}, S R_{u}$ & Time window, Start Time of each $\Delta$ \\
$\mathrm{TL}, \mathrm{EB}, B a$ & Lower bound and upper bound of safety range \\
& Time label, Estimate bolus, \\
$B G_{h}, B G_{l}$, & Basal rate to be checked \\
$\mathrm{CR}, \mathrm{IS}$ & Target high BG, Target low BG, \\
$\mathrm{T}, \mathrm{CI}, B G_{i}$, & Carb ratio, Insulin sensitivity \\
$\mathrm{CE}, \mathrm{FE}$ & Time, Carb input, BG input \\
$\mathrm{AI}, \mathrm{OT}$ & Correction estimate, Food estimate \\
$\mathrm{PN}, \mathrm{Index}$ & Active insulin, Operation type \\
$\mathrm{R}, \mathrm{ST}$ & Pattern name, Index in pattern \\
$\mathrm{D}, \mathrm{TDI}$ & Basal rate, Start time of one rate \\
$\mathrm{TBA}, \mathrm{TBT}$ & Dosage, Total daily insulin \\
$\mathrm{TBD}$ & Temp Basal Amount, Temp Basal Type \\
\hline
\end{tabular}

the definition of $M S E$, we define the safety range $S R$ for bolus dosage and basal rate as follows.

Definition 1:

$S R=\left[S R_{l}, S R_{u}\right]$, where $S R_{l}=Y-2 \sqrt{M S E}$, the $S R_{u}=Y+2 \sqrt{M S E}$, and $Y$ is the regression output for an input vector.

Regardless of the values of bolus dosage and basal rate, we will use the above safety range $S R$ instead.

\subsection{Detection Model 1 for Abnormal Bolus Dosage}

As illustrated in Fig. 1, the bolus doses (blue dotted lines) are discrete. A patient's records can be denoted as a vector: $x=<$ 
$T L(x), E B(x), B G_{h}(x), B G_{l}(x), C R(x), I S(x), C I(x)$, $B G_{i}(x), C E(x), F E(x), A I(x), T(x)>$, representing Time label, Estimate Bolus, Target High BG, Target Low BG, Carb Ratio, Insulin Sensitivity, Carb Input, BG Input, Correction Estimate, Food Estimate, Active Insulin and Time, respectively. For $T L(x)$, we may represent one day as [1-24], [1-12], or [1-8]. For other features, we use the original values from the patient's records. The main types of insulin are bolus and basal. Many patients calculate their estimated bolus using bolus wizard function. Bolus wizard function determines the estimated bolus according to the following rule:

- If $B G_{i}(x)>B G_{h}(x)$,

$$
E B(x)=\frac{B G_{i}(x)-B G_{h}(x)}{I S}+\frac{C I}{C R}-A I(x) ;
$$

- If $B G_{i}(x)<B G_{l}(x)$,

$$
E B(x)=\frac{B G_{i}(x)-B G_{l}(x)}{I S}+\frac{C I}{C R}-A I(x) ;
$$

- Otherwise,

$$
E B(x)=\frac{C I}{C R}-A I(x) .
$$

These rules come from the insulin absorption curve [34] in human body and are embedded in the insulin pump as a bolus wizard function. So a patient needs the pump to help them to calculate an estimated bolus for reference.

To deliver one bolus, a patient has to enter "BG Input" and "Food Estimate" values. Considering "BG Input" as a feature, our scheme has the close-loop properties. Based on the patient pattern, we choose the support vector machine (SVM) [?] regression model to predict bolus dosages. We choose SVM and feature set according to the formula 3, 4, 5 in Section 4.2 and experimental results analysis. After comparisons, we chose SVM. In this paper, we only list and compare the results using linear regression and SVM regression in Section 5. An SVM model is a representation of the examples as points in space, mapped so that the examples of the separate categories are divided by a clear gap that is as wide as possible. New examples are then mapped into that same space and predicted to belong to a category based on which side of the gap they fall on.

In our SVM based design, we select the best hyperplane representing the largest gap between the two classes. Hence, we choose the hyperplane such that the distance from it to the nearest data point on each class is maximized. The optimization problem to maximize the margin with a kernel trick is formulated as follows:

$$
\begin{aligned}
& \min \left\{\frac{1}{2} w^{T} w+C \sum_{i=1}^{l} \xi_{i}\right\} \\
& \text { subject to: } q_{i}\left(w^{T} \varphi\left(p_{i}\right)+b\right) \geq 1-\xi_{i}, \xi_{i} \geq 0 .
\end{aligned}
$$

where $q_{i}$ is either 1 or -1 , indicating the class to which the point $p_{i}$ belongs. Each $p_{i}$ is a $n$-dimensional real vector. The training vector $p_{i}$ is mapped into a higher dimensional space by the function $\varphi$. Then the SVM finds

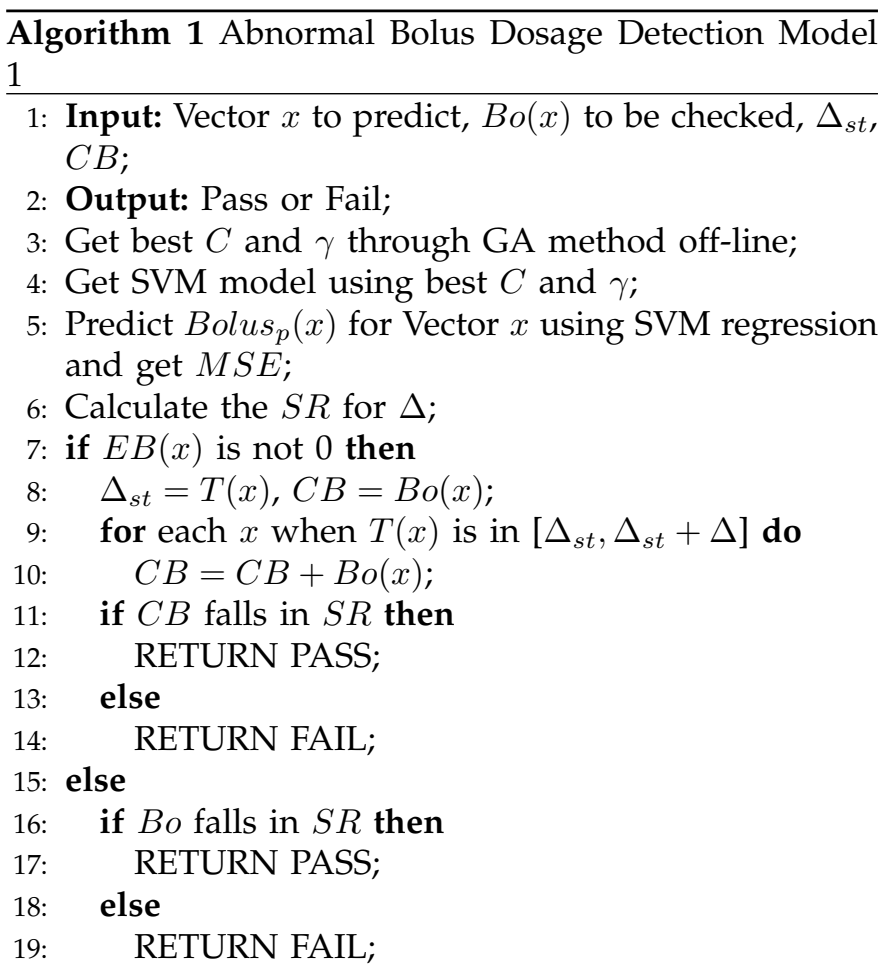

a linear separating hyperplane with the maximal margin in this higher dimensional space. $C(>0)$ is the penalty parameter of the error term. In equation (6), $w$ is also in the transformed space, and $w=\sum_{i} a_{i} q_{i} \varphi\left(p_{i}\right)$. Dot products with $w$ for classification can again be computed by the kernel trick, i.e., $w \bullet \varphi(p)=\sum_{i} a_{i} q_{i} k\left(p_{i}, p_{j}\right)$. Hence, once we obtain $C$ and $\gamma$ that maximize the margin, we obtain the SVM of normal behavior. The kernel function $k\left(p_{i}, p_{j}\right)=\varphi\left(p_{i}\right)^{T} \varphi\left(p_{j}\right)$. In our work, we use a radial basis function as the kernel function: $k\left(p_{i}, p_{j}\right)=\exp \left(-\gamma\left\|p_{i}-p_{j}\right\|^{2}\right), \gamma>0$.

The use of SVM requires the user-defined penalty parameter $C$ for error and kernel specific parameters $\gamma$.

We use a genetic algorithm (GA) ([23]) to get the optimal $C$ and $\gamma$. After we obtain the optimal parameters (i.e., the best model), we test it using additional data and get $M S E$. After having the $M S E$ and the $Y=$ Bolus $_{r}$ for an input vector $x$, we can calculate the safety range $S R$ of bolus dosage within a time window $\Delta$. Then, we check whether $E B(x)$ is 0 or not. If "No", we record the $T(x)$ as $\Delta_{s t}$ and initiate $C B=B o(x)$. Then we check each $x$ when $T(x)$ is in $\left[\Delta_{s t}, \Delta_{s t}+\Delta\right]$, we update the cumulative bolus dosage $C B$ from the start time of $\Delta_{s t}$ by adding the bolus dosage $B o(x)$. If the updated $C B$ falls out of $S R$, it is an abnormal bolus dosage and an alarm will be sent to the patient. Otherwise, it is considered as a normal bolus dosage. If $E B(x)$ is 0 , we check whether $B o$ falls in $S R$. If "Yes", it is a normal bolus dosage. Otherwise, it is an abnormal bolus dosage. The detection model is presented in Algorithm 1. 


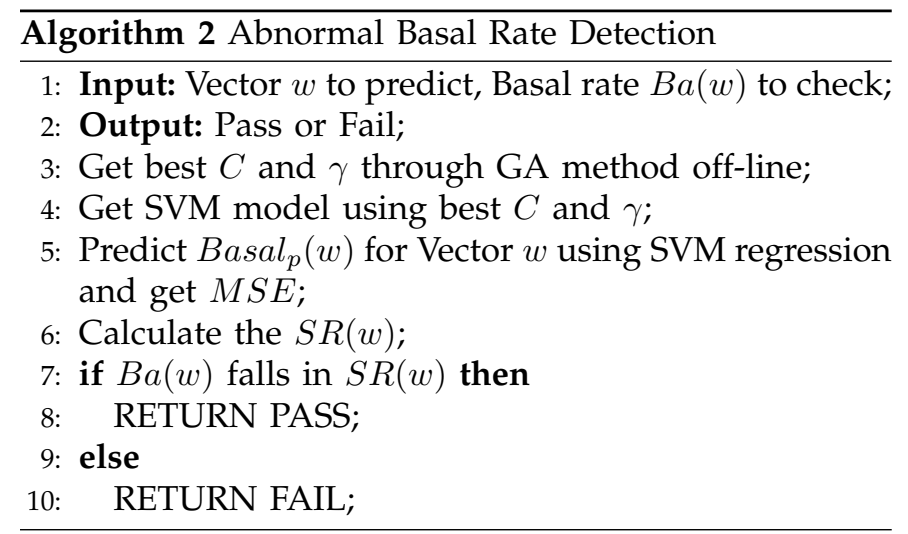

\subsection{Detection Model for Abnormal Basal Rate}

As we can see in Fig. 1, the basal rates (black solid line) are slightly different during different time periods of the day. In addition, the basal rate follows the rules in Section 3.2. Because the basal rate within one day is a piecewise function, we choose the SVM to predict the basal rate. For basal rate prediction, we only needs a small record set that includes all the patterns since the patterns are seldom changed. Here, we slightly change our model showed in [25]. We add Temp (temporary) Basal Amount, Temp Basal Type, Temp Basal Duration as features. Temp Basal Amount represents the ratio of it to the initial setting. The Temp Basal Duration represents the time the temporary basal rate will last. The data can be denoted as a vector: $w=<$ $T L(w), P N(w), \operatorname{Index}(w), R(w), S T(w), T B A(w)$, $T B T(w), T B D(w)>$, representing Time label, Pattern Name, Index, Rate, and Start Time, Temp Basal Amount, Temp Basal Type, Temp Basal Duration, respectively. Regarding $T L(w)$, we label the Time according to the time interval of the pattern. For example, if the time is $1: 00 \mathrm{pm}$ and it falls in the fifth interval of the pattern; we label the time as 5. $S T(w)$ should be divided by 3600000 , which changes its unit from millisecond to hour. We use the generic algorithm to get optimal parameters and $M S E$. After we obtain the $M S E$ at the testing phase and the $Y=\operatorname{Basal}_{r}(w)$ at the detection phase, we can calculate the safety range $S R(w)$ of basal rate at this time. If the basal rate $B a(w)$ to be checked falls out of the $S R(w)$, it is an abnormal basal rate, and we will send an alarm to the patient. Otherwise, it is considered as a normal basal rate. We present our model in Algorithm 2.

\subsection{Daily Total Insulin Dosage Monitoring Model}

Before we design the detection scheme, we have verified that the total insulin dose follows the normal (Gaussian) distribution by Shapiro-wilk test. Thus, we can determine the normal total daily insulin dose region according to the properties of Gaussian distribution. For example, for the confidence of $99.7 \%$, the safety range of total daily insulin dose is $[E-3 \sigma, E+3 \sigma]$, where $E$ is the mean of the total daily insulin dose in 3 months,

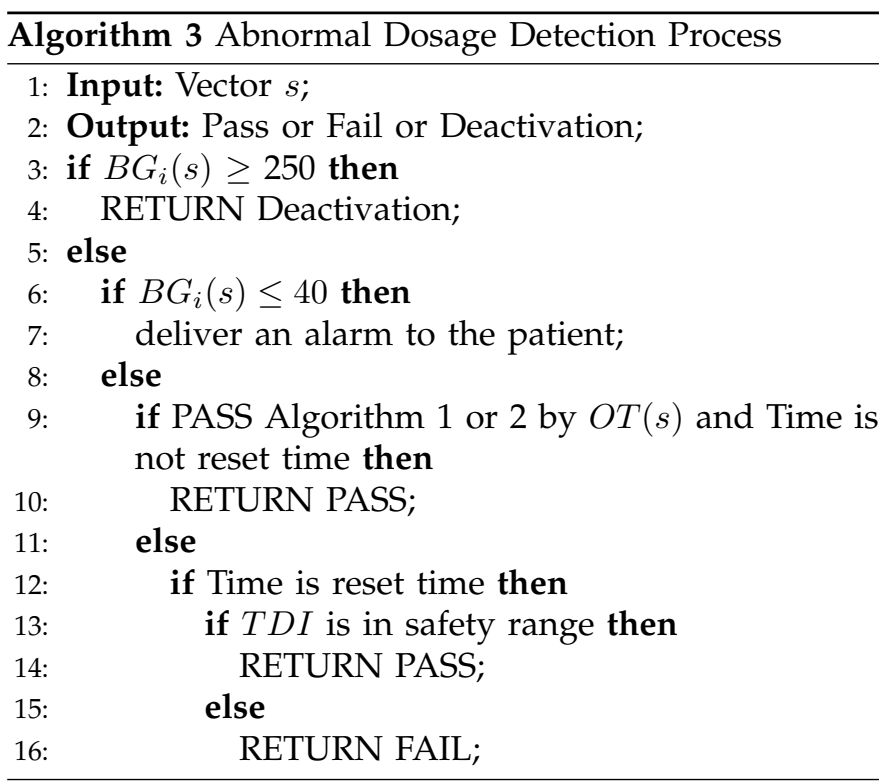

and $\sigma$ is the standard deviation of the total daily insulin dose in 3 months.

\subsection{Combining The Three Models Together}

To combine the three models together, we use a vector $s=<O T(s), T(s), \Delta(s), D(s), T D I(s)>$.

$O T(s), T(s), \Delta(s), D(s)$, and $T D I(s)$ represent Operation Type, Time, Time Interval, Dosage, and Total Daily Insulin, respectively. Operation Type includes bolus or basal, and Time is the event time. $\Delta$ is a fixed time window. Dosage is the actual dosage. Total Daily Insulin is the actual value. If the $B G_{i}$ is higher than $250(\mathrm{mg} / \mathrm{dl})$, it is an emergency requiring deactivation PIPAC scheme. If the $B G_{i}$ is lower than $40(\mathrm{mg} / \mathrm{dl})$, deliver an alarm to the patient. Otherwise, choose either Algorithm 1 or 2 by the Operation Type $O T(s)$. After this, we check the $T D I$ every 24 hours (at personal reset time). Algorithm 3 implements this scheme.

\subsection{Abnormal Bolus Detection Model 2}

All the thresholds in this subsection are determined through data analysis or data mining. Different patients have different thresholds.

As discussed in Section 3.4. the features that we used are: Date, Time, Bolus Volume Selected $(B o)$, Bolus Type $(B T)$, Estimate Bolus $(E B)$, Insulin Sensitivity $(I S)$. Then we have vector $u=<$ $D(u), T(u), B o(u), B T(u), E B(x)>. T h_{\text {total }}$ represents the threshold of total bolus adjustment amount in a day. $T h_{d}$ represents the threshold of bolus adjustment range per dosage, which depends on Insulin Sensitivity $(I S)$ of the patient. $T h_{\text {times }}$ represents the threshold of total adjustment in a small time window $\Delta$. The larger the $I S$, the larger $T h_{d}$, and $T h_{\text {total }} . T h_{\text {times }}=2$ or 3 .

At the beginning of the day, we set the total bolus adjustment amount in a day (representing as Total $_{D i f}$ ) to 0 , the total number of adjustment event (representing 


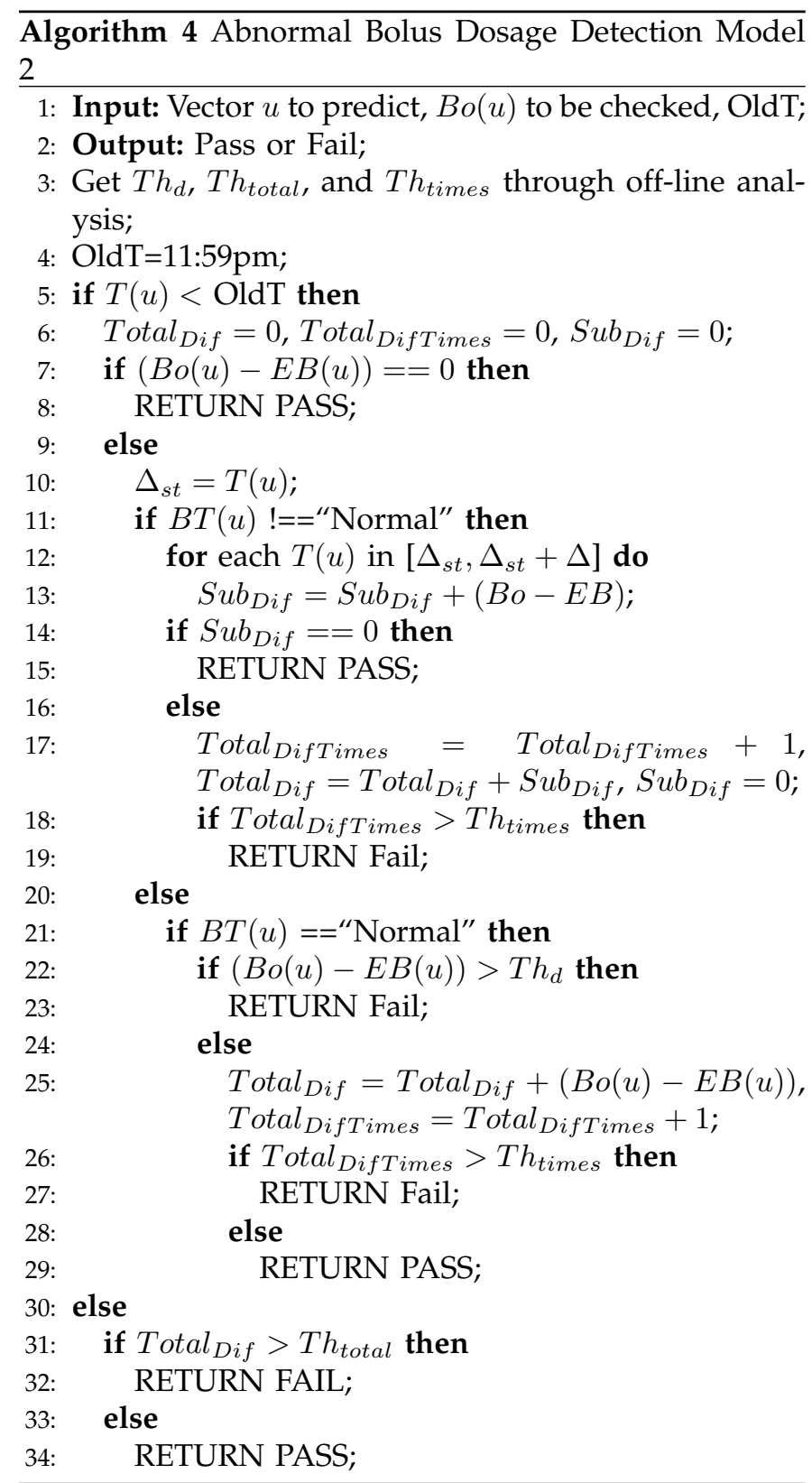

as Total $\left._{\text {DifTimes }}\right)$ to 0 , and the total bolus adjustment amount in a small time window (representing as $S u b_{D i f}$ ) to 0 .

If $B T(u) !=$ "Normal", check whether $S u b_{D i f}$ is 0 . If "YES", it is safe. Otherwise, the total number of adjustment event increases by 1 and the total bolus adjustment amount increases by $S u b_{D i f}$, then reset the $S u b_{D i f}$ to 0 .

If $B T(u)==$ "Normal", check whether $(B o(u)-$ $E B(u))>T h_{d}$. If "YES", it is unsafe. Otherwise, check whether the number of $(B o(u)-E B(u))$ is $0>T h_{\text {times }}$. If "Yes", it is unsafe. Otherwise, it is safe. At the end of the day, we check whether the total bolus adjustment amount is less than the $T h_{\text {total }}$. If "YES", it is safe. Otherwise, it is unsafe and an alarm is sent to the patient. Algorithm 4 implements this scheme.
TABLE 2

Bolus dosage test results using non-linear SVM regression

\begin{tabular}{|c|c|c|c|c|c|}
\hline $\begin{array}{c}\text { Add missing } \\
\text { data? }\end{array}$ & Time label & best $C$ & best $\gamma$ & $M S E$ & $S C C$ \\
\hline Yes & 48 & 5.4062 & 0.0009 & 0.0006 & 0.9990 \\
\hline Yes & 12 & 6.9513 & 0.0134 & 0.0022 & 0.9988 \\
\hline Yes & 8 & 44.05 & 0.0029 & 0.0011 & 0.9995 \\
\hline No & 48 & 3.5472 & 0.0334 & 0.0079 & 0.9953 \\
\hline No & 12 & 9.43 & 0.1345 & 0.0407 & 0.9758 \\
\hline No & 8 & 7.65 & 0.014 & 0.0033 & 0.9980 \\
\hline
\end{tabular}

\section{Performance evaluation}

We conduct experiments using real patient data to evaluate the performance of our scheme.

\subsection{Experimental Setup for Support Vector Ma- chines}

SVM is a form of supervised learning, which provides an effective way to predict bolus dosage and basal rate. In this work, we design an efficient dosage prediction scheme using multiple SVMs. The use of SVMs requires setting user-defined parameters such as $C$, type of kernel, and $\gamma$. The $S C C$ and $M S E$ values were compared to choose a suitable time label methods. In our experiment, we choose the radial basis function as the kernel function. In addition, we use a GA in combination with $k$ fold $(k=5)$ cross validation scheme [24] to get the optimal parameters $C$ and $\gamma$ for a non-linear SVM regression using kernel function. After obtaining the best model using the optimal parameters, we test it using additional data. All computations were carried out using a desktop computer with $2.6 \mathrm{~GB}$ of RAM and a $2.27 \mathrm{GHz}$ of Intel(R) Core(TM) 2 Duo CPU.

\subsection{Experiments for Abnormal Bolus Dosage Detec- tion}

5.2.1 Experimental Results of Bolus Abnormal Detection Model 1

In our experiments, we first preprocess the patient's records. The total sample size of each patient varies. It is close to 500 . We use $80 \%$ of the samples to train the SVM model, and the remaining $20 \%$ to test it. After we use a GA to get the optimal parameters $C$ and $\gamma$, we use them to obtain the optimal SVM model for each patient. Then we test it. Table 2 shows the best parameters and the test results including the $M S E$ and $S C C$ for patient A. We then use a linear SVM model to repeat our experiments after we obtain the best $C$.

Table 2 lists the $M S E$ and $S C C$ of patient A using the linear SVM regression scheme. Comparing Table 2 and 3 , we can see that a non-linear SVM is more suitable for Bolus dosage prediction because the MSE of a nonlinear SVM is smaller than a linear SVM. In addition, the real time labeled as [1-48] within a day gives a better result for patient A. We choose the non-linear SVM to 
TABLE 3

Bolus dosage test results using linear SVM regression

\begin{tabular}{|c|c|c|c|}
\hline Add missing data? & Time label & $M S E$ & $S C C$ \\
\hline Yes & 48 & 0.0022 & 0.9988 \\
\hline Yes & 12 & 0.0198 & 0.9894 \\
\hline Yes & 8 & 0.0280 & 0.9866 \\
\hline No & 48 & 0.0383 & 0.9767 \\
\hline No & 12 & 0.0394 & 0.9761 \\
\hline No & 8 & 0.0409 & 0.9751 \\
\hline
\end{tabular}

TABLE 4

Bolus dosage test results using non-linear SVM regression

\begin{tabular}{|c|c|c|}
\hline Patient label & best $M S E$ & best $S C C$ \\
\hline A & 0.0011 & 0.9874 \\
\hline B & 0.0887 & 0.8976 \\
\hline C & 0.0005 & 0.9983 \\
\hline D & N/A & N/A \\
\hline
\end{tabular}

TABLE 5

Bolus dosage test results using Linear regression

\begin{tabular}{|c|c|}
\hline Patient label & best $M S E$ \\
\hline A & 0.0108 \\
\hline B & 0.0002 \\
\hline C & 0.0105 \\
\hline D & N/A \\
\hline
\end{tabular}

predict the Bolus dosage for patient $\mathrm{A}$, and the best $M S E$ that we get by using the near optimal linear SVM regression is 0.0006 . We find that $\left[\right.$ Bolus $_{p}-2 \sqrt{0.0006}$, Bolus $_{p}+2 \sqrt{0.0006}$ ] is the safety range for that time window $\Delta$. Recall that $S C C=70 \%$ to $100 \%$ is considered as a strong relationship. In our scheme, the best $S C C$ is greater than $99 \%$. This means that we can use the SVM regression to predict a patient's bolus dosage in real time, according to their previous bolus dosage pattern. We also repeat these experiments for other patients. Table 4 shows the results. Table 5 shows the test results using linear regression.

\subsubsection{Experimental Results of Bolus Abnormal Detec- tion Model 2}

This model achieves the similar results as the non-linear SVM regression method. At the same time, it saves a lot of computation and memory. We don't need to get the best parameters using the GA method offline, while we can do a simple data mining process to get the three thresholds. Table 6 shows the test results using bolus abnormal dosage detection model 2. We can see that model 2 can achieve similar $M S E$ level as non-linear SVM regression method while keeping low cost. Thus, we prefer to the bolus abnormal dosage detection model 2 when the patient applies bolus wizard. If the patient does not have an $E B$ at all, we still chose the non-linear SVM regression model or linear SVM regression model in real time.
TABLE 6

Bolus dosage test results using model 2

\begin{tabular}{|c|c|c|c|c|}
\hline Patient label & $T h_{d}$ & $T h_{\text {total }}$ & $T h_{\text {times }}$ & $M S E$ \\
\hline A & 0.3 & 0.5 & 2 & 0.0000 \\
\hline B & 1.5 & 3 & 2 & 0.0056 \\
\hline C & 0.2 & 0.5 & 1 & 0.000 \\
\hline D & N/A & N/A & N/A & N/A \\
\hline
\end{tabular}

TABLE 7

Basal rate test results using non-linear SVM regression

\begin{tabular}{|c|c|c|c|c|}
\hline Patient label & best $C$ & best $\gamma$ & $M S E$ & $S C C$ \\
\hline Patient A & 83.73 & 26.8 & 0.0004 & 0.9682 \\
\hline Patient B & 25.14 & 705.43 & 0.0001 & 0.9999 \\
\hline Patient C & 74.78 & 967.87 & 0.0003 & 0.9261 \\
\hline Patient D & 34.4 & 5.5 & 0.0001 & 0.9999 \\
\hline
\end{tabular}

TABLE 8

Basal rate test results using linear SVM regression

\begin{tabular}{|c|c|c|}
\hline Patient label & $M S E$ & $S C C$ \\
\hline Patient A & 3.03487 & 0.010829 \\
\hline Patient B & 0.0100 & 1.0000 \\
\hline Patient C & 1.0914 & 0.3744 \\
\hline Patient D & 1.0801 & 0.3909 \\
\hline
\end{tabular}

\subsubsection{Parameter Update Policy}

Our scheme can monitor the "Raw-Type" data in logs and capture changed settings. If there is no configuration change to insulin sensitivity, carb ratio, target low BG and target high BG, the SVM regression model is adjusted every 90 days to handle patient dynamics. A subset of the previous 90-day history is used for training, and the new regression is used for the next 90-day interval. After the adjustment, the corresponding parameters $C$ and $\gamma$ are also changed. When the patient is sick, the parameter adjustment cycle can be changed from 90 days to one week.

\subsection{Experiments for Abnormal Basal Rate Detection}

\subsubsection{Experimental Results}

In our experiments, we first preprocessed the patients' records. The total sample size is about 600 for each patient. We use $80 \%$ of the samples to train the SVM model, and the remaining $20 \%$ to test it. We use a similar approach as the previous subsection. For the patient A, the best $C=83.73$ and the best $\gamma=26.8$. After we obtain the best model using the optimal parameters, we run tests. Table 6 shows the best parameters and test results including the $M S E$ and $S C C$ for four patients. We then use a linear SVM model to repeat the experiments after we get the best $C$.

Table 7 lists the $M S E$ and $S C C$ of 4 patients using the non-linear SVM regression scheme. Table 8 lists the $M S E$ and $S C C$ of 4 patients using the linear SVM regression scheme. Table 9 lists the MSE and SCC of 4 patients using the linear regression scheme. Comparing 
TABLE 9

Basal rate test results using Linear regression

\begin{tabular}{|c|c|}
\hline Patient label & best $M S E$ \\
\hline $\mathrm{A}$ & $\mathrm{N} / \mathrm{A}$ \\
\hline $\mathrm{B}$ & 0.2335 \\
\hline $\mathrm{C}$ & 0.0100 \\
\hline $\mathrm{D}$ & 0.0101 \\
\hline
\end{tabular}

TABLE 10

Accuracy of the system of four patients

\begin{tabular}{|c|c|}
\hline Patient label & Accuracy \\
\hline Patient A & $99.43 \%$ \\
\hline Patient B & $99.12 \%$ \\
\hline Patient C & $99.99 \%$ \\
\hline Patient D & $98.89 \%$ \\
\hline
\end{tabular}

Table 7, 8 and 9, we can see that non-linear SVM regression is more suitable for Basal rate prediction. When we use the non-linear SVM to predict the Basal rate, for patient $\mathrm{A}$, the $M S E$ is close to 0.0004 . We determine that $\left[\mathrm{Basal}_{p}-2 \sqrt{0.0004}, \mathrm{Basal}_{p}+2 \sqrt{0.0004}\right]$ is safety range for the Basal rate. In our scheme, the $S C C$ is greater than $95 \%$, indicating that we can use this SVM-based scheme to predict patient basal rate in real time, according to their previous basal rate pattern.

\subsubsection{Parameter Update Policy}

Our scheme can monitor the "Raw-Type" and capture changed settings that have. If the "ChangeBasalProfile" is not actively used, the linear SVM regression is adjusted every 90 days if the patient is not sick. When the patient is sick, the parameter adjustment cycle can be set to one week.

The parameter update policy of the Total Daily Insulin Prediction Model is similar to the one in the previous subsection.

\subsection{Experiments using All Data}

Using the datasets of all patients, we obtain a number of abnormal vectors (including time, bolus dosage, basal rate, etc.) and use these abnormalities to test our PIPAC scheme. Here, we choose the time window $\Delta=15$ mins. Table 10 shows the accuracy of detecting abnormal dosages when we choose the best suitable model for the bolus dosage and basal rate. At last, we use synthetic data (including the real data and abnormal data) to test the algorithm 3 . We test all the synthetic data instead of $20 \%$ testing data in the real data. Less than $2 \%$ dosages in the whole synthetic data were mis-classified. That is the false rejection rate plus the false acceptance rate. There are abnormal data generated by us. It means that there will be at most 1 false alarms every 10 days (we assume 5 dosages per day).

\section{EXTENSION}

\subsection{Communication Range of Wireless Link 5 Tests}

We tested the maximum successful data exchange range. It is $3.45 \mathrm{~m}$, while it ranges from $0.23 \mathrm{~m}-23 \mathrm{~m}$ in the Carelink USB manual. $3.45 \mathrm{~m}$ is a protective communication range when the patient is indoor.

\subsection{Off-line Detection of Settings Change through USB}

When we examine the patient csv logs off-line, we find that logs with "ACTION_REQUESTOR=rf_diagnostic" are related to the USB's application. So we can check the events adjacent to such logs. If the event is related to the setting changes and the patient does not visit the doctors at that time, it is suspicious according to our assumption. This event may be caused by the attackers.

\subsection{Energy Adjustment}

As we can see from Fig. 8, the PC user application needs to detect the signal strength before continuing the communications. So if we adjust the output power, we can limit the access of unauthorized Carelink USB. According to the user manual of Carelink USB, $d=2.3 \sqrt{P}$ holds. Here, $d$ represents transmission distance. If we want to make sure the communication range is $1-3 m$, the maximum output power rating of the transmitter $P$ should be less than $1.701 \mathrm{w}$ by adjusting the resistance.

\section{Discussions}

\subsection{Naive Solution}

A simple public key pair can be applied for authentication over wireless link 5 because each device is certified by the vendor. Both pump and read/controller can have a certificate installed to solve the authentication issue. The problem with this scheme is that there may not be a trusted third party available all of the time. A simple public-key authentication is needed only once to authenticate the pump and the reader/control. All remaining operations can be done with a shared secret via symmetric encryption. A user code can be used as another parameter to set up a shared secret. In the meantime, we can encrypt the wireless control link easily. Another concern is that if every device needs to maintain a public key pair, it is a burden for patients that possess several devices. Also, the patients do not want the vendor (knowing all the $\mathrm{SN}$ ) to have that sort of power and control over their devices and data.

\subsection{Safety Analysis}

Under our scheme, for one patient, the maximum error of Bolus dosage is $2 \sqrt{M S E}$. For patient A, $2 \sqrt{M S E}=$ 0.048 , suppose the total number of safety ranges we counted is 10 in a day, then the total error of insulin is $10 \times 0.048=0.48(\mathrm{u})$. This is less than $1 \mathrm{u}$ and therefore is negligible. For basal rate, the maximum error is $2 \sqrt{M S E}$, 
and the maximum number dose hours that may be administered in one day is 24 . Hence, the total insulin error is $2 \sqrt{M S E} \times 24$. For patient $\mathrm{A}, 2 \sqrt{M S E}=0.04$, hence the total insulin error is $24 \times 0.04=0.96(\mathrm{u})$. It is less than $1 \mathrm{u}$ and is also negligible. In summary, it is safe to use our scheme.

\subsection{Overhead Analysis}

A Medtronic insulin pump operates at 916.5 MHZ. It requires approximately $0.5 \mathrm{~ms}$ to finish the non-linear SVM regression. The energy consumed is negligible compared with ordinary therapy or communication. However, if we use non-linear SVM regression, it may require several minutes to obtain the optimal $C$ and $\gamma$ via the GA method when we update the model every 3 months. From this point of view, the linear SVM regression for bolus prediction still has its advantage. Furthermore, the verification time of our scheme is short, which is very important in regards to the patient's convenience. Our scheme needs to store two small records for Basal rate and Bolus dosage detection. In addition, we need to store the PIPAC program in the insulin pump. All the storage requirements are acceptable given the computing resources of today's insulin pumps.

\subsection{Energy Consumption}

For wireless insulin pump $d=2.3 \sqrt{P}$ holds. Here, $d$ represents transmission distance. If we want to make sure the communication range is $1 \mathrm{~m}$, the maximum output power rating of the transmitter $P$ should be less than 0.189 w by adjusting the resistance. The $P$ is lowered significantly.

\subsection{Security Analysis}

\subsubsection{Defending Against the First Attack}

The first attack is to deliver one large overdose in one shot. Since the upper bound of $S R$ is $Y+2 \sqrt{M S E}$, and the $2 \sqrt{M S E}=0.048$ is far less than $1 \mathrm{u}$, it is impossible to deliver (in one shot) a dose $1 \mathrm{u}$ larger than the estimated dosage. Hence, we can defend against this attack. Since the error of BG measurements is far less than insulin sensitivity, according to Equations (3)(4), the error of calculated insulin is small, then we ignore it.

\subsubsection{Defending Against the Second Attack}

If the attacker gradually increases the dose over a period of several days, our system can still defend against this attack. First, $B G_{i}$ is one of the features being monitored. If the attack happens, $B G_{i}$ will be lowered. Correspondingly, the predicted bolus $S R$ will be decreased. Hence, the attack will be detected due to the detection of bolus (or basal rate) out-of-range. Second, we monitor the cumulative bolus dosage $C B$ within a time window. It is impossible for an attacker to deliver total bolus greater than $S R_{u}$ unless $B G_{i}$ is greater than $250 \mathrm{mg} / \mathrm{dl}$. For basal rate, this kind of attack does not affect the total insulin a lot. Third, our scheme verifies the $T D I$ daily. A suspicious dose can be identified if the TDI falls out of the corresponding safety range. The patient can then check the history log and discover the attack.

\subsubsection{Defending Against the Radcliffe's Attack}

We can monitor: (1) the BG reading from the sensor; and (2) patient's $B G_{i}$ input. As the BG testing technology may have some errors, we use the following approach: if the difference between (1) and (2) is more than $20 \%$, then we consider that there may be intercepting attack between the sensor and the pump. The above approach cannot defend against the Radcliffe's attack $100 \%$ but can mitigate it.

\subsection{Emergency Situations}

It is an orthogonal problem to allow easy access to medical devices when emergencies arise. Many researchers suggested utilizing open access operated by clinical staff during emergencies, e.g., in [12], [13], and [15]. To handle an emergency situation, we can deactivate the PIPAC scheme. Some literatures (e.g. in [17] and [19]) focus on the emergency case. Also since a large dose has a high probability of causing hypoglycemia, doctors and patients try to avoid this from happening. For a patient with elevated body mass, the maximum dose may be set to a larger dose. These patients' safety ranges are also set to a larger value. If the patient becomes hypoglycemic, our scheme issues an alarm to the patient, and the patient can have an emergency food ration that is high in sugar to relieve this situation. What's more, in emergency situations, i.e. the BG is over $250(\mathrm{mg} / \mathrm{dl})$ or lower than $50(\mathrm{mg} / \mathrm{dl})$, the safety range will vary accordingly because our scheme is an online prediction scheme rather than a classification scheme. Thus, our PIPAC scheme can cover this case. When the expected dose is larger than the maximal dose limit, the doctor can change the settings. Also, the patients can split a large dose into several small doses. We observe this method in the patients' medical records. Even though, in this paper we still deactivate the PIPAC scheme to allow open access to wireless insulin pumps.

\section{Related WORK}

A hacker showed how to deliver a 80-volt shock to an ICD [37]. Using an easily obtained USB device, Radcliffe [9] was able to capture data transmitted from the computer and control the insulin pump's operations. Barnaby Jack was able to deliver fatal doses to diabetic patients [6]. Thankfully this attack was only hypothetical and did not result in any actual deaths. Literature [8] [28] propose a traditional cryptographic solution (rolling code) and body-coupled communication to protect the wireless link. However, Jack's attack exploits a vulnerability between the Carelink USB and the pump, neither of which can utilize body-coupled communication. Paper [11] establishes a safety-assured implementation 
of Patient-Controlled Analgesic insulin pump software based on the generic PCA reference model provided by the U.S. FDA. Paper [36] builds a generic insulin infusion pump model architecture and presents a has corresponding hazard analysis document to help later software design. Paper [35] identifies a set of safety requirement that can be formally verified against pump software. Measurements in paper [29] show that in free air, intentional EMI under $10 \mathrm{~W}$ can inhibit pacing and induce defibrillation shocks at distances up to $1-2 \mathrm{~m}$ on implantable cardiac electronic devices.

There are also many solutions proposed to address the security issues of IMDs during non-emergency situations. Literatures [10], [30] and [31] tried to design and develop energy-aware security techniques to reduce the induced energy overhead. The authors of [30] proposed a lightweight security protocol based on a static secret key implemented on ultra-low power ASIC. Authors of [12], [13] and [18] proposed using an additional external device. However, these external devices may become stolen, lost, or forgotten by the patient. The device also discloses the patient's status. Most importantly, this kind of solution adds another device that must be managed by the patient, making it an inconvenient solution for patients, especially when diabetic patients already have to wear many devices. Certificatebased approaches have been proposed in [14], but it requires the device to access the Internet and verify certificates. Rasmussen et al. proposed allowing IMDs to emit an audible alert when engaging in a transaction [15]. However, this approach may consume scarce power resources. Our previous work [16] proposed to utilize the patient's IMD access pattern and designs a novel SVM-based scheme to address the resource depletion attack. It uses a classification scheme rather than the regression scheme used here. It is very effective in nonemergency situations. In another previous work [17], we proposed a novel Biometric-Based two-level Secure Access Control scheme for IMDs when the patient is in emergency situations (such as a coma). Literature [25] proposed a novel patient infusion patterns based access control scheme (PIPAC) for wireless insulin pumps. This scheme employs a supervised learning approach to learn normal patient infusion patterns and calculates a safety range for the total dose in a time window. Then, it detects the abnormal infusions using safety range. The proposed algorithm is evaluated with real insulin pump logs used by several patients for up to 6 months. The evaluation results demonstrate that our scheme can reliably detect the single overdose attack with a success rate up to $98 \%$ and defend against the chronic overdose attack with a very high success rate. Our book [26] gave several defense methods for the wireless insulin pump systems. Our new paper [27] proposed a new near field communication base access control scheme for wireless medical device systems. [20] proposed using friendly jamming to prevent an adversarial access to IMDs. In addition, literature [21] deals with jamming attacks, which can be used to handle the Radcliffe's attack in our paper. Literatures [22] [32] [33] focus on security of health care systems.

\section{Conclusions}

For wireless insulin pump systems, there are two kinds of harmful attacks that are related to dosages, and the vulnerability comes from no authentication on wireless link 5. In this paper, we proposed a PIP based access control scheme that can defend against these attacks. Our scheme leverages the patient dosage history to generate several detection models, and then we determined the safety ranges for each input vector. We employed real patient data to test our scheme, and the results show that our scheme works well and exhibits good performance. Our scheme can be generalized to other infusion systems as well.

\section{REFERENCES}

[1] "2007 national diabetes fact sheet," http://www.cdc.gov/diabetes/pubs/pdf/ndfs 2011.pdf.

[2] "US healthcare equipment and supplies - diabetes." http:/ / www.research.hsbc.com.

[3] "Insulin pumps - global pipeline analysis, opportunity assessment and market forecasts to 2016, GlobalData," http:/ / www.globaldata.com.

[4] http://www.tudiabetes.org/forum/topics/more-interesting-factson.

[5] FDA, “Medical Devices: Infusion Pumps," 2010, http:/ / www.fda.gov/infusionpumps.

[6] http://www.theregister.co.uk/2011/10/27/fatal_insulin_pump_attack.

[7] National Diabetes Information Clearinghouse, http://www.diabetes.niddk.nih.gov/dm/pubs/hypoglycemia/index.aspx.

[8] C. Li, A. Raghunathan and N. K. Jha, "Hijacking an Insulin Pump: Security Attacks and Defenses for a Diabetes Therapy System", in Proc. of the 13th IEEE Intl. Conf. on e-Health NAS, pp. 150-156, 2011.

[9] J, Radcliffe. https:/ / media.blackhat.com/bh-us-11/Radcliffe/BH_US_11 _Radcliff_Hacking_Medical_Devices_WP.pdf

[10] D. Halperin, T. S. Heydt-Benjamin, B. Ransford, S. S. Clark, B. Defend, W. Morgan, K. Fu, T. Kohno, and W. H. Maisel, "Pacemakers and implantable cardiac defibrillators: software radio attacks and zero-power defenses," in Proc. of the 2008 IEEE Symp. on SP'08, pp. 129-142, 2008

[11] B. G. Kim, A. Ayoub, O. Sokolsky, I. Lee, P. Jones, Y. Zhang, and R. Jetley, "Safety-Assured Development of the GPCA Infusion Pump Software," in Proc. of the Intl. Conf. on EMSOFT'11, Taipei, 2011.

[12] P. Inchingolo, S. Bergamasco, and M. Bon, "Medical data protection with a new generation of hardware authentication tokens," in Proc. of Mediterranean Conf. on Medical and Biological Engineering and Computing, 2001.

[13] T. Denning, K. Fu, and T. Kohno, "Absence makes the heart grow fonder: new directions for implantable medical device security," in Proc. of the 3rd Conf. on Hot topics on security, pp. 1-7, 2008.

[14] E. Freudenthal, R. Spring, and L. Estevez, "Practical techniques for limiting disclosure of RF-equipped medical devices," in Proc. of Engineering in Medicine and Biology Workshop, pp. 82-85, 2007.

[15] K. B. Rasmussen, C. Castelluccia, T. Heydt-benjamin, S. Capkun, "Proximity-based access control for implantable medical devices," in Proc. of ACM CCS '09, pp. 410-419, 2009.

[16] X. Hei, X. Du, J. Wu, and F. Hu, "Defending Resource Depletion Attacks on Implantable Medical Devices," in Proc. of IEEE Globecom'10, pp. 1-5, 2010.

[17] X. Hei and X. Du, "Biometric-based Two-level Secure Access Control for Implantable Medical Devices during Emergencies," in Proc. of IEEE INFOCOM'11, pp. 346-350, 2011. 
[18] S. Gollakota, H. Hassanieh, B. Ransford, D. Katabi, and K. Fu, "They can hear your heartbeats: Non-invasive security for implantable medical devices," in Proc. ACM Conf. SIGCOMM'11, pp. 2-13, 2011.

[19] J. Sun, X. Zhu, C. Zhang, and Y. Fang, "HCPP: Cryptography Based Secure EHR System for Patient Privacy and Emergency Healthcare," in Proc. of ICDCS'11, pp. 373-382, 2011.

[20] F. Xu, Z. Qin, C. C. Tan, B. Wang, and Q. Li, "IMDGuard: Securing implantable medical devices with the external wearable guardian," In Proc. of IEEE INFOCOM'11, Shanghai, China, Apr. 2011.

[21] C. Popper, M. Strasser, and S. Capkun, "Jamming-resistant broadcast communication without shared keys," In Proc. of USENIX Security Symp.'09, 2009.

[22] O. Chipara, C. Lu, T. C. Bailey, and G. Roman, "Reliable Clinical Monitoring using Wireless Sensor Networks: Experience in a Stepdown Hospital Unit," in Proc. of ACM Conf. on SenSys'10, Zurich, Switzerland, 2010.

[23] http://en.wikipedia.org/wiki/Genetic_algorithm.

[24] http://en.wikipedia.org/wiki/Cross-validation_(statistics).

[25] X. Hei, X. Du, S. Lin, and I. Lee, "PIPAC: Patient Infusion Pattern based Access Control Scheme for Wireless Insulin Pump System," in Proc. of IEEE INFOCOM 2013, Turin, Italy, Apr. 2013.

[26] X. Hei and X. Du, "Emerging Security Issues in Wireless Implantable Medical Devices", Springer, 2013.

[27] X. Hei, X. Du, and S. Lin, "Poster: Near Field Communication based Access Control for Wireless Medical Devices", in Proc. of ACM MobiHoC 2014.

[28] Ch. Li, "System design and verification methodologies for secure computing", PhD Thesis, 2012.

[29] D. F. Kune, J. Backes, S. S. Clark, D. B. Kramer, M. R. Reynolds, K. Fu, Y. Kim, and W. Xu, "Ghost Talk: Mitigating EMI Signal Injection Attacks against Analog Sensors", in Proc. of the 34th IEEE Symp. on SP'13, pp. 1-15, May. 2013.

[30] S. Hosseini-Khayat, "A lightweight security protocol for ultralow power asic implementation for wireless implantable medical devices", in Proc. of IEEE ISMICT'11, pp. 6-9, 2011.

[31] C. Beck, D. Masny, W. Geiselmann, and G. Bretthauer, "Block Cipher Based Security for Severely Resource-constrained Implantable Medical Devices", in Proc. of ACM ISABEL'11, pp. 62:1-62:5, 2011.

[32] Q. Pan, P. Yang, R. Zhang, C. Lin, S. Gong, L. Li, J. Yan, and G. Ning, "A mobile health system design for home and community use," in Proc of BHI'12, 2012, pp. 116-119.

[33] R. H. Wouhaybi, M. D. Yarvis, P. Muse, C.-Y. Wan, S. Sharma, S. Prasad, L. Durham, R. Sahni, R. Norton, M. Curry, H. Jimison, R. Harper, and R. Lowe, "A Context-Management Framework for Telemedicine: An Emergency Medicine Case Study," in Proc. of Wireless Health'10, pp. 167-173, 2010.

[34] E. D. Lehmann, C. Tarłn, J. Bondia, E. Teufel, and T. Deutsch, “ Incorporating a generic model of subcutaneous insulin absorption into the AIDA v4 diabetes simulator: 2. preliminary bench testing," J. Diabetes Sci. Technol., vol.1, no.5, pp.780-93, 2007.

[35] R. Jetley and P. Jones, "Safety Requirements based Analysis of Infusion Pump Software," in Proc. of the Workshop on Software and Systems for Medical Devices and Services, pp. 21-24, 2007.

[36] Y. Zhang, P. Jones and R. Jetley, "A Hazard Analysis for a Generic Insulin Infusion Pump," J. of Diabetes Sci. and Technol., vol. 4, no. 2, pp. 263-283, 2010.

[37] http://www.computerworld.com/article/2492453/malwarevulnerabilities/pacemaker-hack-can-deliver-deadly-830-voltjolt.html.

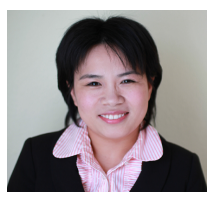

Xiali Hei is a currently assistant professor with the Department of Computer Science and Information Technologies at Frostburg State University. She received the M.S degree from Tsinghua University in 2005, and the Ph.D degree from Temple University in 2014, respectively. Her research interests include wireless network security, security of implantable medical devices and security in cloud computing. She is a Technical Program Committee (TPC) member of premier IEEE conference GLOBECOM, ICC, CyberC,

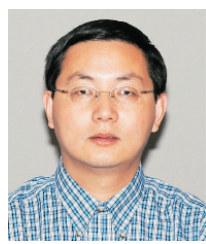

Dr. Xiaojing (James) Du is currently an associate professor in the Department of Computer and Information Sciences at Temple University. $\mathrm{He}$ received his $\mathrm{Ph} . \mathrm{D}$. degree in electrical engineering from the University of Maryland College Park in 2003. He was an Assistant Professor in the Department of Computer Science at North Dakota State University between 2004-2009, where he received the Excellence in Research Award in May 2009. His research interests are security, cloud computing, wireless networks, computer networks and systems. He has published over 120 journal and conference papers in these areas. He has been awarded more than $\$ 3 \mathrm{M}$ research grants from the US National Science Foundation, Army Research Office, Air Force Research Lab, NASA, the Commonwealth of Pennsylvania, and Amazon. He serves on the editorial boards of four international journals. He will serve as the Lead Chair of the Communication and Information Security Symposium of the IEEE ICC 2015, and a Co-Chair of the Mobile and Wireless Networks Track of the IEEE WCNC 2015. He was the Chair of the Computer and Network Security Symposium of the IEEE/ACM International Wireless Communication and Mobile Computing conference $2006-2010$. He is (was) a Technical Program Committee (TPC) member of several premier ACM/IEEE conferences such as INFOCOM (2007 - 2015), IM, NOMS, ICC, GLOBECOM, WCNC, BroadNet, and IPCCC. Dr. Du is a Senior Member of IEEE and a Life Member of ACM.

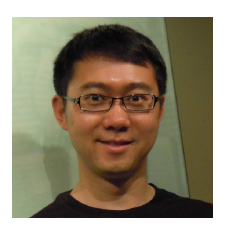

Shan Lin is an assistant professor with the Department of Electrical and Computer Engineering in Stony Brook University. He received his $\mathrm{PhD}$ in computer science at the University of Virginia. His research is in the area of networked systems, with an emphasis on feedback control based design in cyber physical systems. He works on wireless network protocols, medical devices, first responder systems, and smart transportation systems. $\mathrm{He}$ is a member of the IEEE.

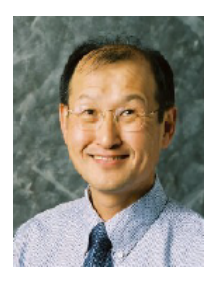

Insup Lee is Cecilia Fitler Moore Professor of Computer and Information Science and Director of PRECISE Center at the University of Pennsylvania. He also holds a secondary appointment in the Department of Electrical and Systems Engineering. He received the Ph.D. in Computer Science from the University of Wisconsin, Madison. His research interests include cyber physical systems (CPS), real-time embedded systems, formal methods and tools, high-confidence medical device systems, and software engineering. He has served on many program committees and chaired many international conferences and workshops. He has also served on various steering and advisory committees of technical societies, including CPSWeek, ESWeek, ACM SIGBED, IEEE TC-RTS, RV, ATVA. He has served on the editorial boards of the several scientific journals and is a founding co-Editor-in-Chief of KIISE Journal of Computing Science and Engineering (JCSE). He was Chair of IEEE Computer Society Technical Committee on Real-Time Systems (2003-2004) and an IEEE CS Distinguished Visitor Speaker (2004-2006). He was a member of Technical Advisory Group (TAG) of Presidents Council of Advisors on Science and Technology (PCAST) Networking and Information Technology (NIT), 2006-2007. He is IEEE fellow and received IEEE TC-RTS Outstanding Technical Achievement and Leadership Award in 2008.

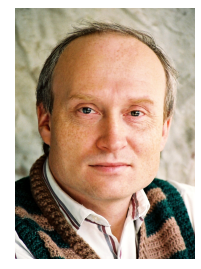

Oleg Sokolsky is a Research Associate Professor of Computer and Information Science at the University of Pennsylvania. His research interests include the application of formal methods to the development of cyber-physical systems, architecture modeling and analysis, specificationbased monitoring, as well as software safety certification. He received his Ph.D. in Computer Science from Stony Brook University. 